\title{
5. Summary
}

1. Different lines of hexaploid oats ( $A$. sterilis- $A$. sativa group) were found to differ from each other by chromosome rearrangements.

2. Five hybrid combinations between $A$. sterilis lines and one sterilisbyzantina combination were examined. All hybrids showed formation of 1-2 quadrivalents indicating translocation differences between lines.

3. These chromosomal differences did not affect the fertility of the hybrids, nor reproductively isolate the various forms.

4. It is proposed that chromosomal variation is an integral part of the genetic system of polyploid oats and one of the means enabling rapid evolution in the group of weeds and cultivated cereals.

Acknowledgments.-The author is greatly indebted to the Agricultural Research Service of the United States Department of Agriculture for a research grant (FG-IS-244) which supported this study.

\section{ReFERENGES}

holden, J. H. W. 1966. Species relationships in Avenae. Chromosoma, 20, 75-124.

IMAM, A. G., AND ALLARD, R. w. 1965. Population studies in predominantly self-pollinated species. VI. Genetic variability between and within natural populations of wild oats from differing habitats in California. Genetics, 51, 49-62.

LADIZINSKY, G., AND zOHARY, D. 1968. Genetic relationships between diploids and tetraploids in the series Eubarbatae of Avena. Can. J. Genet. Cytol., 10, 68-81.

MARENAH, L. J., AND holden, J. H. W. 1967. Karyotype study in Avena. I. The Karyotype of Avena sativa cultivar Condor. Chromosoma, 22, 456-464.

Rajhathy, T. 1963. A standard karyotype for Avena sativa. Can. F. Genet. Cytol., 5, 127-132.

RILEY, R., AND KEMPANNA, C. 1963. The homoeologous nature of non-homologous meiotic pairing in Triticum aestivum deficient for chromosome V (5B). Heredity, 18, 287-306.

tabata, J., AND nishiyama, I. 1966. Cytogenetic study in Avena XV. Chromosome pairing in tetraploid hybrids. Can. 7. Genet. Cytol., 8, 300-305.

zOHARY, D. 1965. Colonizer species in the wheat group. In the Genetics of Colonizer Species. Ed. H. G. Baker and G. L. Stebbins, 404-420. Academic Press, N.Y.

\section{NOTES ON DR SNELL'S OBSERVATIONS CONCERNING THE H-2 LOCUS POLYMORPHISM}

\author{
PETER HULL \\ Biology Department, Strathclyde University
}

Received 28.i.69

\section{INTRODUCTION}

SNell (1968) examined the possible adaptive significance of membrane polymorphism and compared viral oncogenesis, molecular mimicry, rate of virus penetration of cells depending on virus and host genotype, maternalfoetal interaction and heterozygous advantage and other possibilities as mechanisms which could maintain histocompatibility polymorphism and concludes that, theoretically, certain of these types of selection could function in this way while others could not. 


\section{Analysis}

Considering a species of bacterium or virus, certain genotypes of which are able to replicate in a mammalian host, either because their surface antigens mimic those of a host genotype and are thus protected from antibody production, or because they are able to obtain preferential access to host cells of some genotype, Snell concludes that the advantage that a mammalian population would derive from a polymorphism of the mimicked loci would not itself stabilise polymorphism.

TABLE 1

Frequency of host/parasite contacts after one generation of random mating and infection

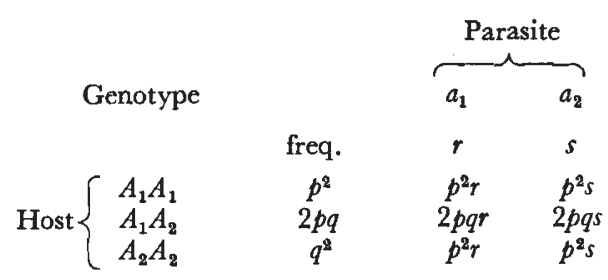

If there are two alleles ( $A_{1}$ and $A_{2}$ with frequencies $p$ and $q$ ) at a locus $A$ in a diploid host species and two alleles $\left(a_{1}\right.$ and $a_{2}$ with frequencies $r$ and $\left.s\right)$ at a mimicking locus in a haploid parasitic species and we consider the case of large populations of host and parasite, in which all those host individuals infected die and in which the parasite can multiply only within the host, then after one generation of random mating and infection, we have the frequencies of host/parasite contacts shown in table 1 . In this case the relative surviving proportions of the three host genotypes would be: $A_{1} A_{1}$ $=p^{2} s: A_{1} A_{2}=0: A_{2} A_{2}=q^{2} r$.

We now have the change in gene frequency, in one generation, of $A_{1}$ :

$$
\left.\begin{array}{l}
\Delta p=\frac{p^{2} s}{p^{2} s+q^{2} r}-p \\
\Delta r=\frac{\left(1-q^{2}\right) r}{\left(1-q^{2}\right) r+\left(1-p^{2}\right) s}-r
\end{array}\right\}
$$

Letting $\Delta p=\Delta r=0$ we have $\hat{p} \hat{s}=q \hat{r}$ and $\hat{q}=\hat{p}$, i.e. $\hat{p}=q=r=\hat{s}=\frac{1}{2}$ as expected. We also have:

$$
A=\left(\begin{array}{l}
\frac{\partial \Delta p}{\partial p} \frac{\partial \Delta p}{\partial r} \\
\frac{\partial \Delta r}{\partial p} \frac{\partial \Delta r}{\partial r}
\end{array}\right)_{p=r=\frac{1}{2}}=\left(\begin{array}{ll}
+1 & -1 \\
+\frac{2}{3} & 0
\end{array}\right)
$$

with latent roots $=\frac{-1 \pm \sqrt{1-8 / 3}}{-2}$, indicating as Snell concluded that there is no stable gene frequency equilibrium, but further that the gene frequencies of both host and parasite will change in a series of oscillations of increasing magnitude. An examination of $A$ shows that if $p$ and $r$ are both slightly above (or both slightly below) equilibrium, $p$ being the further from 0.5 , then $p$ will move towards 0.5 and $r$ away from 0.5 till $p$ reaches 0.5 and $r$ 
is further from 0.5 than $p$ was originally. Then $r$ will move towards the value of $p$, which will continue to move away on the opposite side of 0.5 till $r$ overshoots $p$ by a still greater amount. Thus $r$ will always reach 0 or 1 before $p$ does so.

This is true for several modifications of the simple basic assumptions, such as (i) that only a proportion $(m)$ of the susceptible host genotypes come into contact with the parasite, or (ii) that infection frequency is a function of the rarity of the various genotypes so that, for example, the relative surviving proportions of the three host genotypes might be:

$$
A_{1} A_{1}=p^{2} e^{-m r}: A_{1} A_{2}=2 p q e^{-m}: A_{2} A_{2}=q^{2} e^{-m s} \text {. }
$$

If we examine what would happen in a large population where this type of selection is taking place, using a computer to calculate from $\Delta p$ and $\Delta r$ the gene frequency $(p)$ of allele $A_{1}$ in the host and allele $a_{1}$ in the parasite $(r)$ in succeeding generations we obtain some set of results such as are plotted in fig. 1. In this case the mean number of parasites $(m)$ per host is one, and the initial frequencies are $p=0.5$ and $r=0.55$. It is apparent that at some stage (in this case between generations 30 and 35), the frequency of one allele in the parasite becomes very low: in this case $s=0.0001$, and fixation would probably take place in a population of finite size. There is then a rapid change towards fixation of allele $A_{2}$ in the host and the consequent elimination of the parasite. However, mutation from $a_{1}$ to $a_{2}$ would cause further oscillations at this stage. Also the occasional interbreeding of individuals from two neighbouring populations, differing by chance in the alleles approaching fixation in a species like the mouse whose breeding population is said to be divided into isolated demes, could re-establish a population with intermediate histocompatibility gene frequencies.

It has been assumed so far that the potentially antigenic surface of the parasite is a product of its own genotype. If, however, the virus surface is composed of host cell membrane, as has been suggested for certain viruses by Franklin (1958) on the basis of chemical analysis and electron microscope photographs, e.g. Morgan et al. (1956), possibly bearing the host histocompatibility specificities (Nandi, 1967), this situation could also lead to an unstable gene frequency equilibrium in the host. If we start with a population with gene frequency very close to the unstable equilibrium point of $p=\frac{1}{2}$, where by chance after selection one of the homozygotes is present in slight excess $(d)$ we have the relative genotypic frequencies $A_{1} A_{1}=\frac{1}{2}+d$ : $A_{1} A_{2}=0: A_{2} A_{2}=\frac{1}{2}-d$. The proportion of viruses bearing both types of surface antigen is not relevant in this situation if they could infect only heterozygous hosts. The relative fitnesses are $A_{1} A_{1}=1-\left(\frac{1}{2}-d\right)$ and $A_{2} A_{2}=1$ $-\left(\frac{1}{2}+d\right)$. Then the proportion of $A_{1} A_{1}$ individuals in the next generation will be:

$$
A_{1} A_{1}^{\prime}=\frac{\left(\frac{1}{2}+d\right)^{2}\left[1-\left(\frac{1}{2}-d\right)\right]}{\left(\frac{1}{2}+d\right)^{3}+\left(\frac{1}{2}-d\right)^{3}}
$$

so that in this generation:

$$
-d^{\prime}=\frac{1}{2}-\frac{\left(\frac{1}{2}+d\right)^{3}}{\left(\frac{1}{2}+d\right)^{3}+\left(\frac{1}{2}-d\right)^{3}}=-3 d .
$$

Again there is no reason why one allele should be favoured rather than the other and in a subdivided or partly subdivided population different alleles 


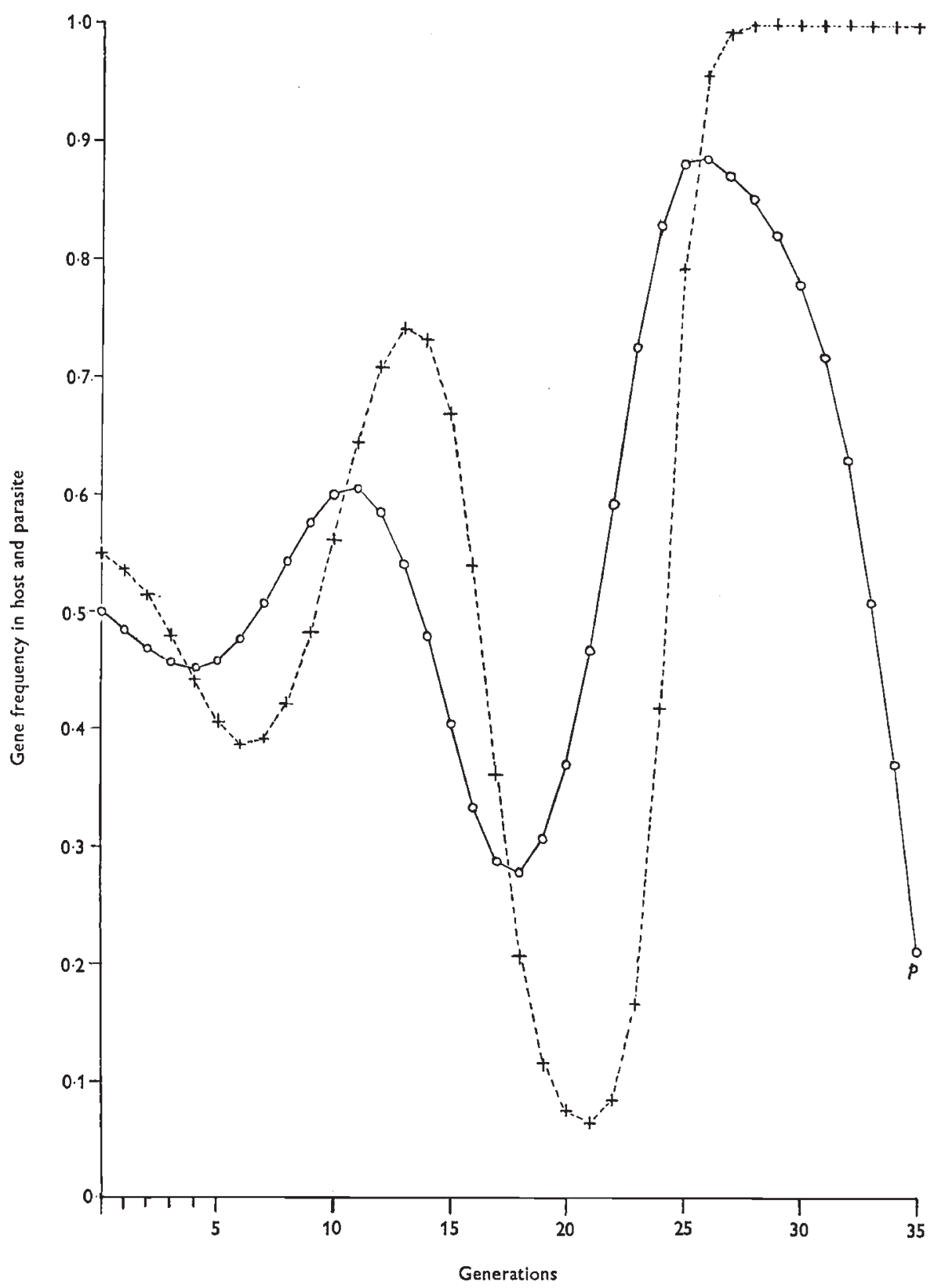

FIG. 1.-Gene frequency in host and parasite during 35 generations of selection where the parasite shows molecular mimicry. The frequency of allele $A_{1}$ in the host is $p$ and of $a_{1}$ in the parasite is $r$.

could reach high frequencies in different regions, re-establishing a population with intermediate frequencies by interbreeding.

Maternal-foetal interaction (autoincompatibility) could maintain a stable polymorphism involving many alleles at a locus, rather than just two or three, and could account for the multiallele polymorphism found in the 
case of several histocompatibility loci, provided that selective elimination of homozygotes of each allele is greater than elimination of all heterozygotes involving that allele, and that females discriminate against offspring of their own genotype (Hull, 1966).

It would appear that none of these mechanisms suggested by Snell can be discounted completely on theoretical grounds as being responsible for the mixture of histocompatibility genotypes which is inferred to exist in mammalian populations. More data on the actual distribution of histocompatibility type between and within families would be necessary to decide between these hypotheses.

\section{Summary}

Of the mechanisms suggested by Snell as being responsible for the maintenance of histocompatibility differences in mammalian populations, none of those which was examined theoretically could be discounted completely as a possible cause.

\section{ReFERENCES}

FRANKLIN, R. M. 1958. An hypothesis to explain the relation between the synthesis and release of animal viruses from infected cells and the lipid content of the viruses. Experientia, 14, 346-348.

HULL, P. 1966. Possible stability of polymorphism in a multiallele incompatibility system in the mouse. Genetics, 54, 1049-1053.

MORGAN, c. et al. 1956. Structure and development of viruses observed in the electron microscope. J. Exp. Med., 104, 171-182.

NANDi, s. 1967. The H-2 locus and susceptibility to Bittner virus borne by red blood cells in the mouse. P.N.A.S., 58, 485-492.

SNELL, G. D. 1968. The H-2 locus of the mouse: observations and speculations concerning its comparative genetics and polymorphism. Folia Biologica, 14, 335-358.

\section{HETEROGENEITY OF MITOCHONDRIA IN THE INTERSPECIFIC HYBRID MESOCRICETUS NEWTONI $\times$ M. AURATUS}

P. RAICU, B. VLADESCU, I. BORŞAN and S. STAICU

Department of Genetics, University of Bucharest, and Department of Genetics, Institute of Biology, Roumania

Received 26.i.69

\section{INTRODUGTION}

THE occurrence of mixed mitochondrial populations in the cells, demonstrated cytochemically for the succinate-dehydrogenase and cytochromeoxidase in "petites" strains of Saccharomyces (Avers et al., 1965) raises the question of the complexity of the ways by which the uniformity of the cytoplasmic determinants is accomplished, either following the production of mutated genetic factors within the intracellular mitochondrial population, or following the combination by hybridisation of two different mitochondrial populations.

Another remarkable example of mitochondrial heterogeneity has been described in maize (McDaniel and Sarkissian, 1966, 1968), which could be 